\title{
HIF-1a overexpression in adipose mesenchymal stem cell-derived exosomes ameliorate hypoxia- induced dysfunction and inflammation in HUVECs
}

Xingxing Chen ( $\nabla$ xingxingchen2012@126.com )

Gansu Provincial Hospital

\section{Yonghong Sun}

Gansu Provincial Hospital

Xiaoyan Lei

Gansu Provincial Hospital

Yunshan Cao

Gansu Provincial Hospital

Mingdong Gao

Gansu Provincial Hospital

Junhui Chen

Gansu Provincial Hospital

\section{Baixin Bao}

Gansu University of Traditional Chinese Medicine

Lili Chen

Gansu University of Traditional Chinese Medicine

\section{Research Article}

Keywords: HIF-1a, ADSCs, SIRT3, exosomes, hypoxia, angiogenesis, inflammatory response

Posted Date: August 19th, 2021

DOI: https://doi.org/10.21203/rs.3.rs-794247/v1

License: (1) (1) This work is licensed under a Creative Commons Attribution 4.0 International License. Read Full License 


\section{Abstract \\ Background}

Increasing evidence suggests that ADSCs execute their paracrine function via the secretion of exosomes, especially under hypoxic conditions. However, the mechanisms by which ADSCs-derived exosomes (ADSC-exos) enhance angiogenesis under hypoxia remain unclear.

\section{Methods}

Exosomes were isolated from HIF-1a-modified ADSCs culture supernatants. To investigate the effects HIF-1a-ADSC-exos on HUVECs, cell growth, apoptosis, and tube formation assay were performed with or without HIF-1a-ADSC-exos. Moreover, to determine the function of HIF-1a-ADSC-exos, the therapeutic effects of ADSC-exos and HIF-1a-ADSC-exos were examined in PAH rats.

\section{Results}

Exosomes released by HIF-1a-modified ADSCs rescued the impaired angiogenic ability, migratory function, and inflammatory factors of hypoxia-injured HUVECs, with increased SDF-1a, Rac1, Rac2, VEGF and IL-10 expression. Furthermore, exos-HIF-1a activated SIRT3 to enhance angiogenesis in HUVECs and induced IL-10 expression to inhibit inflammatory response. Block SIRT3 or SDF-1a abolished the angiogenic effect in HUVECs.

\section{Conclusion}

Our findings indicated that the SIRT3 contributed a crucial role in HIF-1a-ADSC-exos in tissue repair under hypoxia.

\section{Introduction}

Pulmonary arterial hypertension (PAH) is a chronic life-threatening disease characterized by progressive pulmonary vascular remodeling and increased pulmonary arterial pressure, resulting in right ventricular overload and subsequent death [1, 2]. Pathological changes of PAH are complex, including thickening of intimal and medial layers, fibrotic vasculopathy, and perivascular inflammatory cell infiltration, lead to a progressive proliferation, migration, or phenotype switches of pulmonary artery smooth muscle cell (PASMC) and pulmonary arterial vascular endothelial cell (PAVEC), and leading to an abnormal elevation in pulmonary arterial pressure [3-5]. Despite the great advances in clinical, functional, and hemodynamic improvement in PAH patients has been achieved in recent years[5], however, the molecular and pathogenetic mechanisms of PAH remain poor. 
Adipose-derived stem cells (ADSCs) are mesenchymal stem cells harvested from adipose tissue (AT) capable of self-renewal and multipotent differentiation into various types of other cells [6, 7]. Enormous attention has been focusing on the ADSCs due to the easy isolating procedure and a higher harvest rate than bone marrow $[8,9]$. Increasing evidence have shown that ADSCs has been proven to be useful for corneal wounds and regenerating non-healing cutaneous, cardiovascular disease [10,11]. Exosomes (exos) are vesicles with a size of 30-200 nm derived from the cell membrane of cells and released to the extracellular environment after fusion of multivesicular endosomes and plasma membranes under normal or pathological conditions [12-14]. Subsequent studies implied that exosomes released from MSCs (MSC-exos) and ADSCs (ADSC-exos) have been revealed to promote spinal cord injury recovery and hepatic recovery, reduce myocardial ischemia/refusion injury and pulmonary hypertension by receptor-ligand binding, direct membrane fusion, and endocytosis [15-17]. Hypoxia-inducible factor 1alpha (HIF-1a), a heterodimeric transcription factor complex consisting of $a$ and $\beta$ subunits, is a key regulator of the cellular adaptation to hypoxia $[18,19]$. HIF-1 a was reported as an important activator of endothelial cells in the injured and adjacent vasculature by mediating a series of metabolic responses to maintain energy balance and redox homeostasis $[20,21]$. Furthermore, ADSCs-exo enhanced the proliferation and migration of HaCaT cells by inducing the phosphorylation of AKT and the expression of HIF-1a to promote wound healing [22]. However, the effects of HIF-1 a modified ADSCs on PAVEC remain unclear.

In this study, we explored the effects of genetic modification ADSCs on the hypoxia-induced PAH. The effects of HIF-1a on ADSCs were determined by cell proliferation and apoptosis under normoxic and hypoxic state. Then the in vitro functions of HIF-1a modified ADSCs-exo modulated HUVECs were explored, as well as the underlying mechanisms. Finally, a PA model was employed to examine the in vivo effects of HIF-1 a modified ADSCs-exo on pulmonary vascular remodeling.

\section{Materials And Methods}

Animal studies

The study was carried out in accordance with the Guidelines for the Care and Use of Laboratory Animals and approved by Animal Care and Use Committee of Gansu Province people's hospital (No. 202004FT). A total of 48 adult male C57BL/6 mice (4weeks) obtained from Shanghai SLAC Laboratory Animal Co.,Ltd (Shanghai, China) were housed under pathogen-free conditions with 12-h light/dark cycle and $22 \pm 1^{\circ} \mathrm{C}$ and a humidity of $40-60 \%$. Experimental PAH Model was performed as previously described [23]. The mice were randomly divided into normal group (saline) and PAH group (exosomes form vehicle, exosomes form HIF-1a). Then $50 \mu$ l exosomes (from $5 \times 10^{7}$ ADSCs) were intravenously injected. The mice in all the groups were killed after intraperitoneal injection with $10 \%$ chloral hydrate, and samples of lung tissues were removed histopathological examinations.

Preparation and characterization of exosomes 
Exosomes were isolated from ADSCs using ExoQuick-TC (SBI, USA) according to the manufacturer's instructions. Western blotting was performed to detect the expression of known exosomal markers antiCD63 (\#ab59479, Abcam, Cambridge, MA, USA) and anti-CD81 (\#ab79559, Abcam) and the exosome morphology were observed by transmission electron microscopy (TEM) (Hitachi, H7500 TEM, Tokyo, Japan).

Adipose-derived stem cell isolation and culture

The groin adipose tissue was harvested from C57BL/ 6 mice ageing 4 weeks, after clearing and $0.075 \%$ collagenase digestion, then centrifugation removed the supernatant and undigested fat. The cells were resuspended in DMEM (Invitrogen, Carlsbad, CA, USA) supplemented with 10\% FBS (Invitrogen) and 1\% penicillin-streptomycin (P/S) at $37^{\circ} \mathrm{C}$ with $5 \% \mathrm{CO}_{2}$. The first change of medium took place $8 \mathrm{~h}$ later and the medium was replaced every $2-3$ days. Cells were digested and passaged after reaching $80 \%$ confluency.

Establishment of HIF-1a stable overexpression ADSCs

Full-length HIF-1a coding sequence was subcloned into the lentiviral vector pCDH-CMV-MCS-EF1-Puro (System Biosciences, Beijing, China). Lentivirus was generated in HEK293T cells and filtration with 0.22 $\mu \mathrm{m}$ filter membrane. To construct the stable cell lines with target gene overexpression, ADSCs were infected with Lentivirus for $24 \mathrm{~h}, 2 \mu \mathrm{g} / \mathrm{mL}$ puromycin (Sigma-Aldrich, St Louis, MO, USA) was added to culture medium after transducing with lentivirus $48 \mathrm{~h}$ later to continuously screen the stable cells for 10 days. The small interfering RNA (siRNA) targeting SIRT3 were obtained from Shanghai GenePharma Co., Ltd (Shanghai, China) and transfected using Lipofectamine 2000 (Invitrogen) in accordance with the manufacturer's instructions. The in vitro preconditioning hypoxia model was established by flooding the chamber with $95 \% \mathrm{~N}_{2}$ and $\mathrm{CO}_{2}$ using a ProOx model 110 oxygen regulator purchased from BioSpherix (New York, NY, USA).

Cell viability assay

Cells of each group were planted in 96-well microculture plates with $1 \times 10^{4} /$ well and cultured at $37^{\circ} \mathrm{C}$ with $5 \% \mathrm{CO}_{2}$ overnight. $10 \mu \mathrm{l}$ of CCK-8 solution (TAKARA, USA) was added to each well and incubated for $2 \mathrm{~h}$ at 24, 48, 72, and $96 \mathrm{~h}$. Finally, absorbance was assessed using a microplate reader (Bia-rad, Hercules, CA, USA) at a wavelength of $450 \mathrm{~nm}$.

Cell apoptosis assay

To evaluate cell apoptosis, apoptotic ratios were evaluated by the Annexin V-FITC/PI Apoptosis Detection Kit according to the manufacturer's recommendations. The stained cells were analyzed were analyzed by FACScan flow cytometer (Becton Dickinson, USA) and evaluated using the ModFit program software.

Evaluation of lung histological pathology and pulmonary vascular permeability 
To assess the severity of lung injury, the lung tissues were harvested and fixed with $4 \%$ paraformaldehyde and embedded in paraffin and then cut into $4 \mu \mathrm{m}$ sections. Then, the sections were deparaffinized and stained with hematoxylin-eosin (H\&E). The degrees of inflammation, congestion, and edema were evaluated using lung injury score as previously described [24].

For arterial blood gas studies, partial pressure of oxygen $\left(\mathrm{PaO}_{2}\right)$ and partial pressure of carbon dioxide $\left(\mathrm{PaCO}_{2}\right)$ were measured from blood samples obtained from the femoral artery. The dry weight was determined, and the lung wet-to-dry weight (W/D) ratio was measured to assess the pulmonary vascular permeability. For hemodynamic studies, the PICCO system was used to evaluate the mean arterial pressure, heart rate, stroke volume variation, and cardiac output.

Measurement of Inflammatory Cytokines

Mouse blood was centrifuged at $4^{\circ} \mathrm{C}$ and $3000 \mathrm{r} / \mathrm{min}$ for 10 minutes, and the serum was harvested to measure the levels of tumor necrosis factor-a (TNF-a), interleukin-10 (IL-10), vascular endothelial growth factor (VEGF) and monocyte chemotactic protein 1 (MCP-1) using ELISA kits in accordance with the manufacturer's instructions (eBioscience, San Diego, CA, USA).

Transwell invasion assays

Invasion assays of HUVECs in vitro were performed using transwell chambers with $8 \mu \mathrm{m}$ pores (Corning Incorporated, Corning, NY, USA) coated with Matrigel (BD Bioscience, Bedford, MA, USA). Cells were harvested after the specific treatment and resuspended in serum-free medium. About $4 \times 10^{5}$ cells in 100 $\mu \mathrm{l}$ serum-free medium was added into the upper chamber, $600 \mu \mathrm{l}$ complete medium was added into lower chamber. After incubation for $24 \mathrm{~h}$, the cells were fixed in $4 \%$ paraformaldehyde for 30 min and stained with $0.1 \%$ crystal violet for $15 \mathrm{~min}$. The invaded cells were imaged and counted in five randomly selected fields at 200x magnification using an Olympus optical microscope (Tokyo, Japan).

Tube formation assay

After reaching $80 \%$ cell confluence, ADSCs cells were cultured with serum-free DMEM for another $24 \mathrm{~h}$, and the supernatant was collected as a conditioned medium. $100 \mu \mathrm{L}$ Matrigel was added into 24-well plates to polymerize for $2 \mathrm{~h}$ at $37^{\circ} \mathrm{C}$. Then $2 \times 10^{5}$ HUVECs were seeded into coated wells with conditioned medium. Images were taken by an Olympus optical microscope $6 \mathrm{~h}$ later, and the numbers of tubes formed were counted to measure tubule-forming ability.

Western Blot Analysis

Total protein was extracted from cells were RIPA buffer (Sigma-Aldrich) containing Protease Inhibitor Cocktail (Cell Signaling Technology, Inc., Beverly, MA, USA). Protein concentration was determined using the bicinchoninic acid (BCA) protein assay kit (Thermofisher, Carlsbad, CA, USA). Equal amounts of protein extracts $(40 \mu \mathrm{g})$ were separated by $10 \%$ SDS-PAGE and transferred to polyvinylidene difluoride membranes (Bia-Rad). Then the membranes were blocked and incubated overnight with rabbit anti-HIF- 
1a (Cell Signaling Technology), rabbit anti-SIRT3 (Cell Signaling Technology), rabbit anti-SDF-1a (Cell Signaling Technology), rabbit anti-VEGF (Cell Signaling Technology), rabbit anti-Rac1 (Proteintech Group. Inc, Rosemont, IL, USA), rabbit anti-Rac2 (Proteintech Group. Inc), and mouse anti-GAPDH (Cell Signaling Technology) antibodies, respectively. The expression levels of protein were measured by enhanced chemiluminescence reagents (Millipore, Plano, TX, USA).

\section{Statistical Analysis}

Data are expressed as means \pm standard deviation (SD). Each experiment was performed independently at least three times. Student's t-test was used to compare the difference between two groups and the difference among multiple groups were determined based on one-way analysis of variance. Statistical analyses were performed using the SPSS 21.0 statistical package (SPSS Inc., Chicago, IL, USA).

\section{Results}

\section{Isolation and identification of the exosomes secreted by ADSCs}

It has been suggested that ADSCs could repair the damaged tissues [6, 7]. Flow cytometry demonstrated that isolated ADSCs were CD24 + CD44 + CD90 + Sca-1 + CD34- CD45- (Fig. 1A). As expected, ADSCs transduced with HIF-1a displayed the increased expression of HIF-1a at protein levels (Fig. 1B). Then we explored the roles of HIF-1a on ADSCs, the results showed that there was no significant change in cell viability and apoptosis under either normoxia or hypoxia state (Fig. 1C and D). It promoted us to consider exos might play the crucial roles in tissue repair due to paracrine functions of stem cells. USC-exos were obtained using ExoQuick-TC and observed under a TEM (Fig. 1E). Western blot showed that ADSCs-exos were positive for specific markers CD63 and CD81 (Fig. 1F). These results suggested that the substances we isolated could be identified as exosomes.

\section{Exosomes from HIF-1a-modified ADSCs enhance proliferation and tube formation in HUVECs under hypoxia state}

In $\mathrm{PAH}$, the alteration of pulmonary artery endothelial cells was the main pathological basis of pulmonary vascular remodeling with pulmonary hypertension. Previous studied have shown that hypoxia induces injury of endothelial cells. Therefore, we next determined whether the secretion of HIF-1a-exos from ADSCs exert biological activity to mediate specific intercellular communication. To explore the potential roles of exosomes in endothelial cells, we first assessed the effect of Vector-exos and HIF-1a-exos on the proliferation and apoptosis of HUVECs under normoxia and hypoxia state. As shown in Fig. 2A, CCK-8 assay showed that HIF-1a-exos treatment significantly increased the proliferation of HUVECs compared with Vector-exos under hypoxia state, but no significant change was observed under normoxia state. Flow cytometry results revealed that HIF-1a-exos decreased the apoptosis rate of HUVECs induced by hypoxia, but there was no significant change between Vector-exos and HIF-1a-exos under normoxia state (Fig. 2B). 
Then we examined the influence of exos on HUVECs tube formation, one important step involved in angiogenesis. The tube formation assay indicated that HIF-1a-exos displayed a better effect on tube formation of HUVECs compared with Vector-exos under hypoxia state (Fig. 2C). All these results suggested that overexpression of HIF-1 a could abolished the injuries of HUVECs induced by hypoxia.

\section{Migratory ability and inflammatory cytokines release of hypoxia-preconditioned HUVECs was rescued by exosomes from HIF-1a-modified ADSCs}

It has been established that migratory ability endothelial cells of HUVECs and inflammatory disorder were involved in the pathological changes of PAH [4]. Therefore, Transwell system was introduced to study how exosomes regulate the migratory ability of HUVECs. As shown in Fig. 3A, hypoxia dramatically impaired the migratory ability of HUVCEs. However, HIF-1a-exos could revere the inhibition of migratory ability under hypoxic conditions. Furthermore, the promotion of hypoxia on IL- 6 and MCP-1 secretion (Fig. 3B) and the repression on IL-10 secretion (Fig. 3C) also could be reversed by HIF-1a-exos treatment in HUVECs. In addition, ELISA showed HIF-1a-exos increased VEGF production in HUVECs both under normoxia and hypoxia state (Fig. 3D). These results showed that HIF-1a-exos could alleviate hypoxiainduced HUVECs injury.

\section{Exosomes from HIF-1a-modified ADSCs alleviated the effects of HUVECs induced by hypoxia via up- regulating SIRT3 expression}

Then we explored the molecular mechanism of exosomes from HIF-1a-modified ADSCs regulated angiogenesis and inflammatory response. Previous studies have shown that SIRT3 regulated multiple physiological processes such as metabolism and aging $[25,26]$. Interestingly, we found that the expression of SIRT3 was significantly increased in HIF-1a-exos compared with Vector-exos treated cells (Fig. 4A, left). The downstream genes of SIRT3 involved in angiogenesis and inflammatory response were also detected, as we expect, the expression levels of SDF-1a, Rac1, Rac2 were decreased, whereas VEGF was increased after HIF-1a-exos treatment (Fig. 4A and 4F). However, SIRT3 silencing reversed the alteration of SDF-1a, Rac1, Rac2 and VEGF induced by HIF-1a-exos (Fig. 4A, right). To investigate the importance of SIRT3 in exosomes from HIF-1a-modified ADSCs in HUVECs functions, siRNA targeting SIRT3 were performed in HUVECs under hypoxic conditions prior to exosome extraction. CCK-8 assay showed that SIRT3 silencing by siRNA remarkably repressed the viability of HUVECs treated with HIF-1aexos under hypoxia state (Fig. 4B). Consistent with the result of CCK-8 assays, the Flow cytometry assays showed that SIRT3 silencing contributed to the apoptosis of HUVECs (Fig. 4C). Similarly, knockdown of SIRT3 abolished the enhancing effect of HIF-1a-exos on tube formation (Fig. 4D) and migratory ability (Fig. 4E) of HUVECs. Furthermore, Elisa results showed that SIRT3 silencing also reversed the effects of HIF-1a-exos on HUVECs, except VEGF expression (Fig. 4F). Taken together, our data indicated that SIRT3 might play a crucial role of effects of HIF-1a-exos on HUVECs.

HIF-1a-modified ADSCs ameliorated PAH phenotypes induced by hypoxia in vivo 
In order to further investigate the efficacy of HIF-1 a-exos in the PAH, PAH models were established in mouse models, which including the preventive model and therapeutic model. After intermittent hypoxia for 4 weeks, RVSP (Fig. 5A), RV/BM (Fig. 5B), representative images of pulmonary arteries stained with H\&E (Fig. 5C), RV/LV +S (Fig. 5D) and remodeling of small pulmonary arteries presented as MT\% (Fig. 5E) and MA\% (Fig. 5F) in normal group were remarkably increased compared with hypoxia group. These results indicated that a PAH model in mouse had been established with remodeling of small pulmonary arteries. By using a hypoxia-induced PAH preventive mouse model, mice that treated with HIF1a-exos attenuated PAH phenotypes, as indicated reductions in RVSP, RV/BM (Fig. 5B), RV/LV + S (Fig. 5D), as well as MT\% (Fig. 5E) and MA\% (Fig. 5F). Taken together, these results indicated that HIF-1aexos improved endothelial function both in vitro and in vivo.

\section{Discussion}

Pulmonary arterial hypertension (PAH), one type of pulmonary hypertension, is a chronic and progressive pulmonary vascular disease associated with a high morbidity and mortality $[1,4]$. It is well known that the features of PAH were mainly pulmonary vascular remodeling, including structural and functional alteration of the smooth muscle cells and endothelial cells and inflammation [27]. Although the approved treatments for PAH have been improved, clinical prognosis of patients remain poor [28]. Accumulating studies have documented that ADSCs were originated from stromal-vascular fragments of adipose tissue and used for therapeutic application due to its pluripotent differentiation and immune tolerance $[6,10]$. In the present study, we observed that exosomes from ADSCs was able to improve hypoxia-induced PAH by regulating endothelial cells disorders and inflammation response. Furthermore, our results suggested that exosomes from HIF-1 a modified ADSCs mediated the inhibition of cell survival and the release of inflammatory factors HUVECs-induced by hypoxia. In addition, we found that SIRT3 was required for HIF1a-exos-mediated endothelial cells disorders and inflammation response.

Previous evidence has shown that HIF-1a could promote tissue repair by upregulating the expression of target genes to increase oxygen delivery to the injured tissue in hypoxia adaptation $[18,29]$. Recent study has shown that HIF-1a-modified ADSCs could recruit an existing endogenous endothelial cell population to induce angiogenesis in a 3D cell construct in vitro [30]. With the spanking development of exosome research, an increasing number of researchers are focusing on exosomes as a potential clinical treatment. Stem cell-derived exosomes provide cell-free therapies as an alternative to traditional stem cell therapies due to immune rejection, low retention rate, low survival rate, and tumor-causing risk of cell transplantation of stem cell [31, 32]. Exosomes derived from the HIF-1 a modified MSCs enhanced the angiogenesis to provide cardioprotection in myocardial infarction [33]. In our present study, HIF-1amodified ADSCs-derived exosomes ameliorated PAH vascular remodeling, reduce the degree of lung fibrosis and right ventricular hypertrophy in vivo. Then HUVECs were used as a model to explore the potential molecular mechanisms of exosomes on endothelial cells in vitro. Our present study demonstrated that HIF-1a-modified ADSCs-derived exosomes could effectively abolished injuries of HUVECs induced by hypoxia. 
Previous studies have shown that SIRT3 was involved in a wide variety of beneficial activities, including anti-oxidation, regulation of energy metabolism, anti-degeneration and anti-tumor activities by modulating ROS generation [34-36]. Recent study has revealed that SIRT3 was reported to inhibit Asbestos-induced Pulmonary Fibrosis [37] and SIRT3 was found to play a crucial role in cardioprotective effects of Resveratrol on RV dysfunction in PAH [38]. It promoted us to detect the expression of SIRT3 in HUVECs treated with HIF-1a-exos. Our results showed that HIF-1a-exos could upregulated the expression of SIRT3 in HUVECs under hypoxia state, accompanying with of SDF-1a, Rac1, Rac2 decreased and VEGF increased. The cell proliferation, apoptosis, tube formation and migration of HUVECs were impeded when deletion of SIRT3. Other inflammatory cytokines were significantly reduced after the addition of stem cellderived exosomes. The results suggested that the suppression of HIF-1a-exos on PAH pulmonary vascular remodeling were associated with up-regulation SIRT3 expression.

\section{Conclusion}

In summary, the present data indicated that HIF-1a-modified ADSCs-derived exosomes could reduce the cell injuries and inflammatory response of HUVECs partly through activating SIRT3 dependent manner, which might offer the mechanism and theoretical basis of HIF-1a-modified ADSCs-mediated tissue repair in PAH.

\section{Abbreviations}

PAH: Pulmonary arterial hypertension

ADSCs: Adipose-derived stem cells

HE: Hematoxylin and eosin

qRT-PCR: Real-time quantitative polymerase chain reaction

PaO2: partial pressure of oxygen

PaC02: partial pressure of carbon dioxide

TNF-a: tumor necrosis factor-a

IL-10: interleukin-10

VEGF: vascular endothelial growth factor

MCP-1: monocyte chemotactic protein 1

HIF-1a-ADSC-exos: HIF-1a-ADSC-derived exosomes

\section{Declarations}




\section{Conflict of interest statement}

All authors declare that they have no known competing financial interests or personal relationships that could have appeared to influence the work reported in this paper.

\section{Acknowledgements}

None.

\section{Funding}

This work was supported by

\section{Contributions}

LXY designed the research, CXX and SYH performed the research; CYS, GMD and CJH performed in vitro and in vivo studies; BBX and CLL analyzed the data; CXX and SYH wrote the manuscript as first authors. All authors read and approved the final manuscript.

\section{Ethics declarations}

Ethics approval and consent to participate

Written informed consents were obtained from all participants and this study was permitted by the Ethics Committee of Gansu Province people's hospital.

\section{Consent for publication}

Not applicable.

\section{Availability of data and materials}

The datasets generated during the current study are available from the corresponding author on reasonable request.

\section{References}

1. Barst RJ. Evaluation and treatment for angina in pulmonary arterial hypertension. AM J MED. 2004;116(6):427-8.

2. Galie N, Saia F, Palazzini M, Manes A, Russo V, Bacchi RM, Dall'Ara G, Monti E, Dardi F, Albini A, et al. Left Main Coronary Artery Compression in Patients With Pulmonary Arterial Hypertension and Angina. J AM COLL CARDIOL. 2017;69(23):2808-17.

3. Lawrie A, Francis SE. Frataxin and endothelial cell senescence in pulmonary hypertension. $J$ CLIN INVEST 2021, 131(11). 
4. Sharifi KD, Kim K, Simon MA. Current Understanding of the Right Ventricle Structure and Function in Pulmonary Arterial Hypertension. FRONT PHYSIOL. 2021;12:641310.

5. Vonk NA, Westerhof BE, Westerhof N. The Relationship Between the Right Ventricle and its Load in Pulmonary Hypertension. J AM COLL CARDIOL. 2017;69(2):236-43.

6. Dai R, Wang Z, Samanipour R, Koo Kl, Kim K. Adipose-Derived Stem Cells for Tissue Engineering and Regenerative Medicine Applications. STEM CELLS INT. 2016;2016:6737345.

7. Trzyna A, Banas-Zabczyk A. Adipose-Derived Stem Cells Secretome and Its Potential Application in "Stem Cell-Free Therapy". Biomolecules 2021, 11(6).

8. An YH, Kim DH, Lee EJ, Lee D, Park MJ, Ko J, Kim DW, Koh J, Hong HS, Son Y, et al. High-Efficient Production of Adipose-Derived Stem Cell (ADSC) Secretome Through Maturation Process and Its Non-scarring Wound Healing Applications. Front Bioeng Biotechnol. 2021;9:681501.

9. Damous LL, de Carvalho A, Nakamuta JS, Shiroma ME, Louzada A, Soares-Jr JM, Krieger JE, Baracat EC. Cell-free therapy with the secretome of adipose tissue-derived stem cells in rats' frozen-thawed ovarian grafts. STEM CELL RES THER. 2018;9(1):323.

10. Suzuki K, Akita S, Yoshimoto H, Ohtsuru A, Hirano A, Yamashita S. Biological Features Implies Potential Use of Autologous Adipose-Derived Stem/Progenitor Cells in Wound Repair and Regenerations for the Patients with Lipodystrophy. INT J MOL SC/2019, 20(21).

11. Zhong Y, Li X, Wang F, Wang S, Wang X, Tian X, Bai S, Miao D, Fan J. Emerging Potential of Exosomes on Adipogenic Differentiation of Mesenchymal Stem Cells. Front Cell Dev Biol. 2021;9:649552.

12. Koh YQ, Almughlliq FB, Vaswani K, Peiris HN, Mitchell MD. Exosome enrichment by ultracentrifugation and size exclusion chromatography. Front Biosci (Landmark Ed). 2018;23:86574.

13. Song D, Yang D, Powell CA, Wang X. Cell-cell communication: old mystery and new opportunity. CELL BIOL TOXICOL. 2019;35(2):89-93.

14. Yu B, Zhang X, Li X. Exosomes derived from mesenchymal stem cells. INT J MOL SCI. 2014;15(3):4142-57.

15. Lai RC, Arslan F, Lee MM, Sze NS, Choo A, Chen TS, Salto-Tellez M, Timmers L, Lee CN, El OR, et al. Exosome secreted by MSC reduces myocardial ischemia/reperfusion injury. STEM CELL RES. 2010;4(3):214-22.

16. Lai RC, Tan SS, Teh BJ, Sze SK, Arslan F, de Kleijn DP, Choo A, Lim SK. Proteolytic Potential of the MSC Exosome Proteome: Implications for an Exosome-Mediated Delivery of Therapeutic Proteasome. Int J Proteomics. 2012;2012:971907.

17. Xin H, Li Y, Cui Y, Yang JJ, Zhang ZG, Chopp M. Systemic administration of exosomes released from mesenchymal stromal cells promote functional recovery and neurovascular plasticity after stroke in rats. J Cereb Blood Flow Metab. 2013;33(11):1711-5.

18. Thangarajah H, Vial IN, Grogan RH, Yao D, Shi Y, Januszyk M, Galiano RD, Chang El, Galvez MG, Glotzbach JP, et al. HIF-1alpha dysfunction in diabetes. CELL CYCLE. 2010;9(1):75-9. 
19. Tirpe AA, Gulei D, Ciortea SM, Crivii C, Berindan-Neagoe I. Hypoxia: Overview on Hypoxia-Mediated Mechanisms with a Focus on the Role of HIF Genes. INT J MOL SCI 2019, 20(24).

20. Shen WC, Liang CJ, Wu VC, Wang SH, Young GH, Lai IR, Chien CL, Wang SM, Wu KD, Chen YL. Endothelial progenitor cells derived from Wharton's jelly of the umbilical cord reduces ischemiainduced hind limb injury in diabetic mice by inducing HIF-1alpha/IL-8 expression. STEM CELLS DEV. 2013;22(9):1408-18.

21. Wang K, Dai X, He J, Yan X, Yang C, Fan X, Sun S, Chen J, Xu J, Deng Z, et al: Endothelial Overexpression of Metallothionein Prevents Diabetes-Induced Impairment in Ischemia Angiogenesis Through Preservation of HIF-1alpha/SDF-1/VEGF Signaling in Endothelial Progenitor Cells. DIABETES 2020, 69(8):1779-1792.

22. Zhang Y, Han F, Gu L, Ji P, Yang X, Liu M, Tao K, Hu D. Correction to: Adipose mesenchymal stem cell exosomes promote wound healing through accelerated keratinocyte migration and proliferation by activating the AKT/HIF-1alpha axis. J MOL HISTOL. 2020;51(4):467.

23. Vazquez-Garza E, Bernal-Ramirez J, Jerjes-Sanchez C, Lozano O, Acuna-Morin E, Vanoye-Tamez M, Ramos-Gonzalez MR, Chapoy-Villanueva H, Perez-Plata L, Sanchez-Trujillo L, et al. Resveratrol Prevents Right Ventricle Remodeling and Dysfunction in Monocrotaline-Induced Pulmonary Arterial Hypertension with a Limited Improvement in the Lung Vasculature. OXID MED CELL LONGEV. 2020;2020:1841527.

24. Vazquez-Garza E, Bernal-Ramirez J, Jerjes-Sanchez C, Lozano O, Acuna-Morin E, Vanoye-Tamez M, Ramos-Gonzalez MR, Chapoy-Villanueva H, Perez-Plata L, Sanchez-Trujillo L, et al. Resveratrol Prevents Right Ventricle Remodeling and Dysfunction in Monocrotaline-Induced Pulmonary Arterial Hypertension with a Limited Improvement in the Lung Vasculature. OXID MED CELL LONGEV. 2020;2020:1841527.

25. Giralt A, Villarroya F. SIRT3, a pivotal actor in mitochondrial functions: metabolism, cell death and aging. BIOCHEM J. 2012;444(1):1-10.

26. Paulin R, Dromparis P, Sutendra G, Gurtu V, Zervopoulos S, Bowers L, Haromy A, Webster L, Provencher $\mathrm{S}$, Bonnet $\mathrm{S}$, et al. Sirtuin 3 deficiency is associated with inhibited mitochondrial function and pulmonary arterial hypertension in rodents and humans. CELL METAB. 2014;20(5):827-39.

27. Schermuly RT, Ghofrani HA, Wilkins MR, Grimminger F. Mechanisms of disease: pulmonary arterial hypertension. NAT REV CARDIOL. 2011;8(8):443-55.

28. Klinger JR, Elliott CG, Levine DJ, Bossone E, Duvall L, Fagan K, Frantsve-Hawley J, Kawut SM, Ryan JJ, Rosenzweig EB, et al: Therapy for Pulmonary Arterial Hypertension in Adults: Update of the CHEST Guideline and Expert Panel Report. CHEST 2019, 155(3):565-586.

29. Guo J, Hu Z, Yan F, Lei S, Li T, Li X, Xu C, Sun B, Pan C, Chen L. Angelica dahurica promoted angiogenesis and accelerated wound healing in $\mathrm{db} / \mathrm{db}$ mice via the HIF-1alpha/PDGF-beta signaling pathway. Free Radic Biol Med. 2020;160:447-57.

30. Est-Witte SE, Farris AL, Tzeng SY, Hutton DL, Gong DH, Calabresi KG, Grayson WL, Green JJ. Non-viral gene delivery of HIF-1alpha promotes angiogenesis in human adipose-derived stem cells. ACTA 
BIOMATER. 2020;113:279-88.

31. Braccioli L, van Velthoven C, Heijnen CJ. Exosomes: a new weapon to treat the central nervous system. MOL NEUROBIOL. 2014;49(1):113-9.

32. Fukumitsu M, Suzuki K. Mesenchymal stem/stromal cell therapy for pulmonary arterial hypertension: Comprehensive review of preclinical studies. J CARDIOL. 2019;74(4):304-12.

33. Sun J, Shen H, Shao L, Teng X, Chen Y, Liu X, Yang Z, Shen Z. HIF-1alpha overexpression in mesenchymal stem cell-derived exosomes mediates cardioprotection in myocardial infarction by enhanced angiogenesis. STEM CELL RES THER. 2020;11(1):373.

34. Allison SJ, Milner J. SIRT3 is pro-apoptotic and participates in distinct basal apoptotic pathways. CELL CYCLE. 2007;6(21):2669-77.

35. Wang Z, Sun R, Wang G, Chen Z, Li Y, Zhao Y, Liu D, Zhao H, Zhang F, Yao J, et al. SIRT3-mediated deacetylation of PRDX3 alleviates mitochondrial oxidative damage and apoptosis induced by intestinal ischemia/reperfusion injury. REDOX BIOL. 2020;28:101343.

36. Wang Z, Sun R, Wang G, Chen Z, Li Y, Zhao Y, Liu D, Zhao H, Zhang F, Yao J, et al. SIRT3-mediated deacetylation of PRDX3 alleviates mitochondrial oxidative damage and apoptosis induced by intestinal ischemia/reperfusion injury. REDOX BIOL. 2020;28:101343.

37. Cheresh P, Kim SJ, Jablonski R, Watanabe S, Lu Z, Chi M, Helmin KA, Gius D, Budinger G, Kamp DW. SIRT3 Overexpression Ameliorates Asbestos-Induced Pulmonary Fibrosis, mt-DNA Damage, and Lung Fibrogenic Monocyte Recruitment. INT J MOL SCI2021, 22(13).

38. Bernal-Ramirez J, Silva-Platas C, Jerjes-Sanchez C, Ramos-Gonzalez MR, Vazquez-Garza E, ChapoyVillanueva H, Ramirez-Rivera A, Zarain-Herzberg A, Garcia N, Garcia-Rivas G. Resveratrol Prevents Right Ventricle Dysfunction, Calcium Mishandling, and Energetic Failure via SIRT3 Stimulation in Pulmonary Arterial Hypertension. OXID MED CELL LONGEV. 2021;2021:9912434.

\section{Figures}



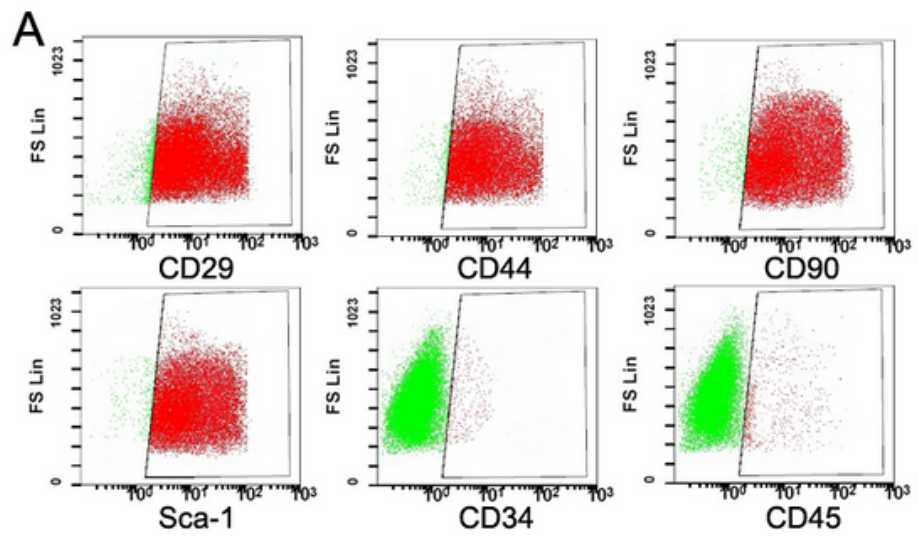

B
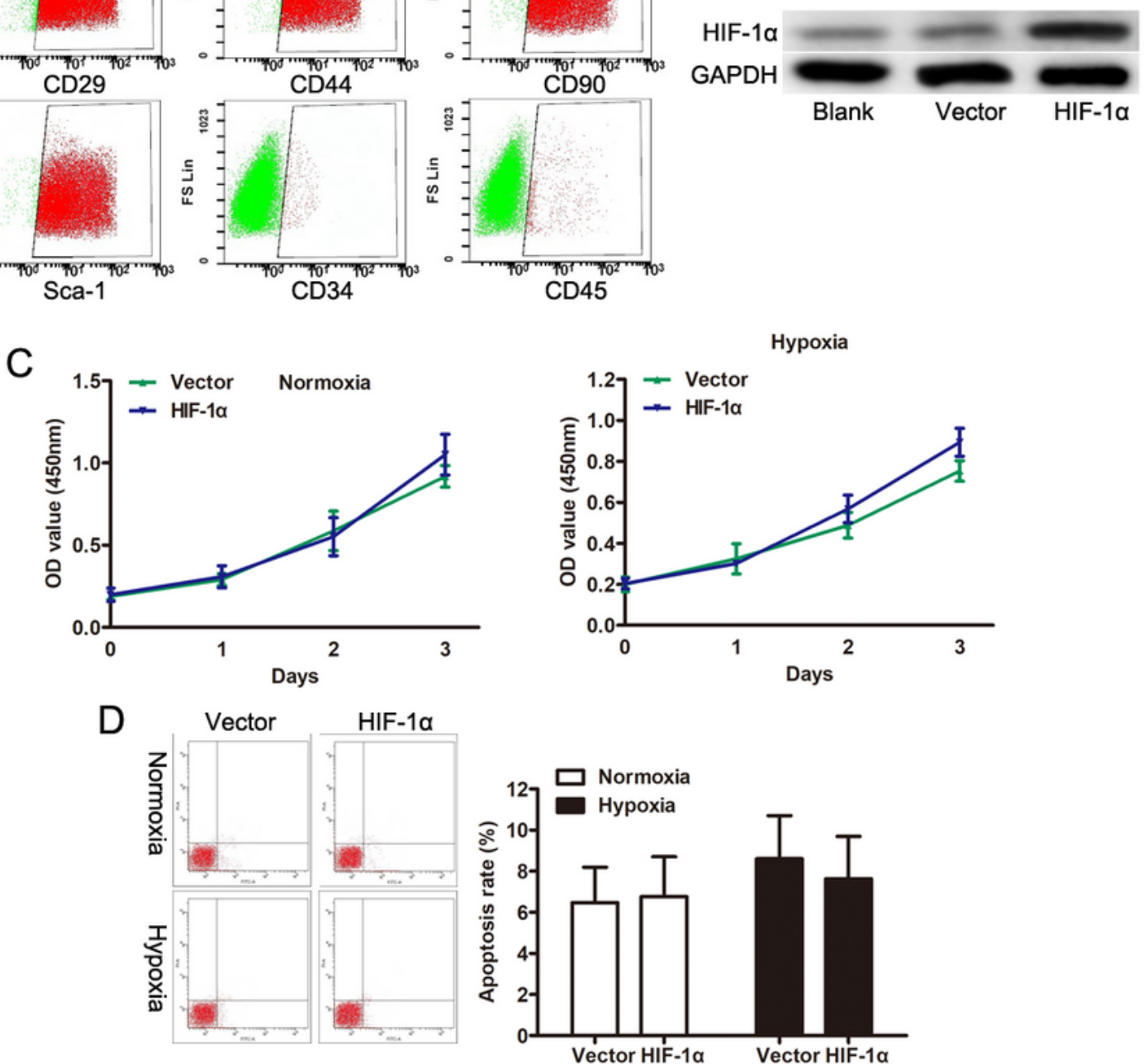

$\mathrm{E}$
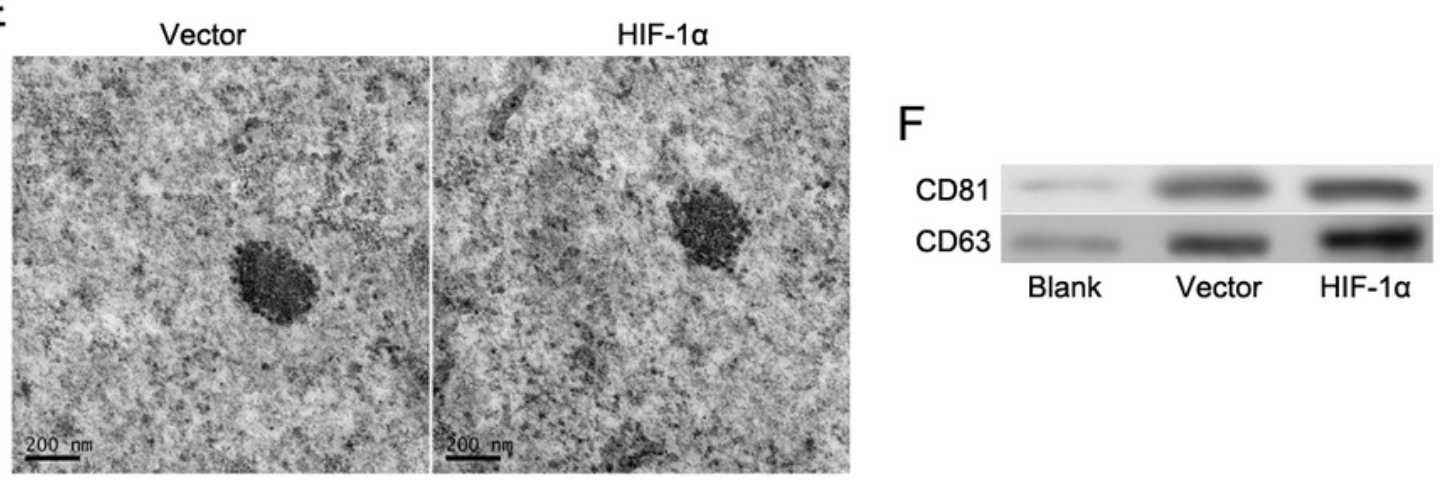

\section{Figure 1}

Characterization of ADSC-derived exosomes. (A) Characterize the stem cell surface markers of ADSCs by flow cytometric analysis. (B) The expression of HIF-1 a proteins were evaluated by Western blotting analysis after HIF-1a overexpression in ADSCs. (C) Cell viability was assessed in ADSCs with or without HIF-1a overexpression by CCK-8. (D) Apoptosis level was assessed in ADSCs with or without HIF-1a overexpression by CCK-8. (E) Representative images showing the morphology of ADSC-derived exosomes 
by transmission electron microscopy (scale bar = $200 \mathrm{~nm}$ ). (F) Expression of exosomal markers (CD81 and CD63) examined by western blot analysis.
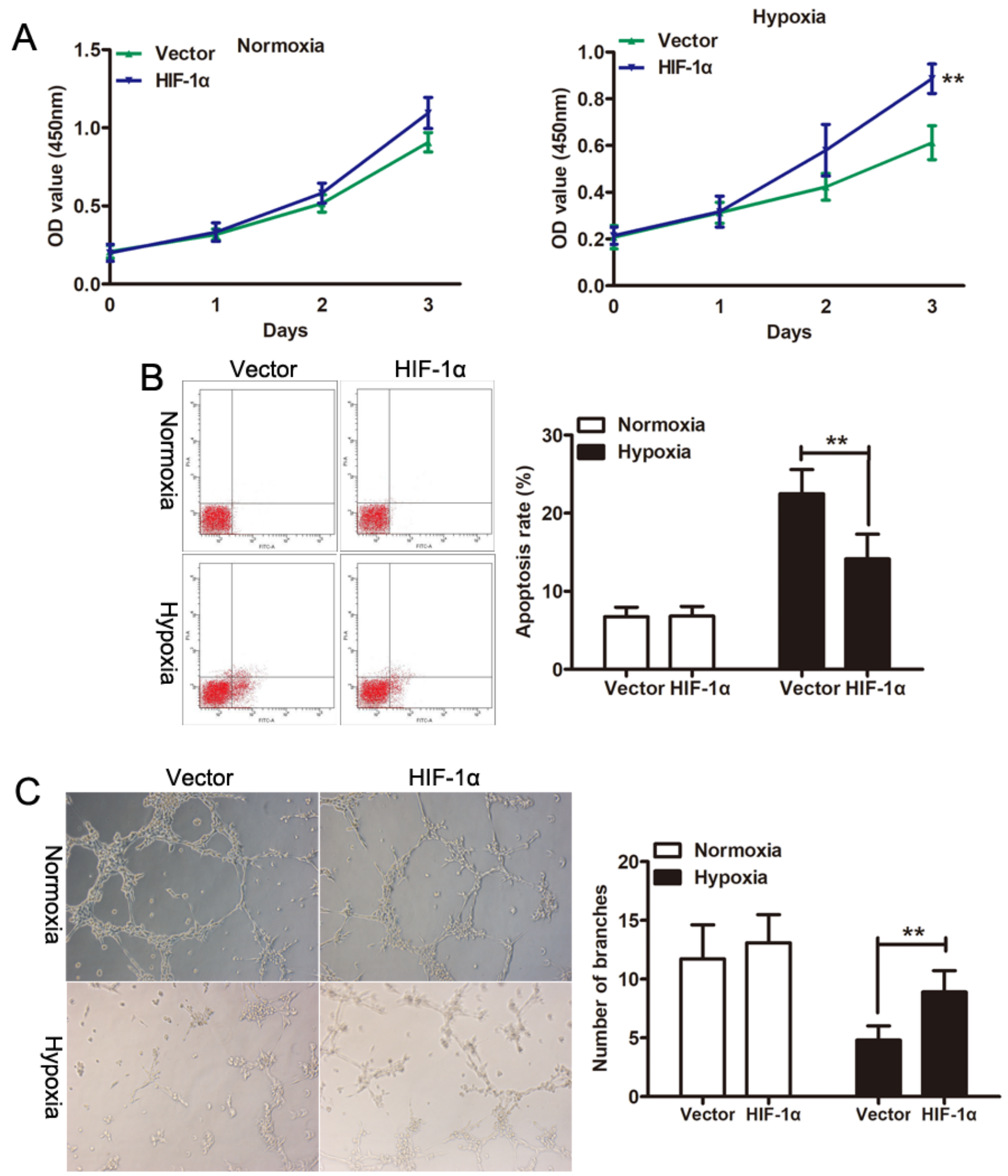

Figure 2

Effects of exos-HIF-1a on the viability, apoptosis, and tube formation of HUVECs under hypoxia state. (A) The viability of HUVECs was determined by CCK-8 assay after treatment with Exos-Vector or Exos-HIF-1a under normoxia or hypoxia state. (B) Apoptosis level was determined in HUVECs treated with Exos-Vector 
or exos-HIF-1 a under normoxia or hypoxia state. (C) Tube formation assay in HUVECs treated with ExosVector or Exos-HIF-1a under normoxia or hypoxia state. **P $<0.01$.
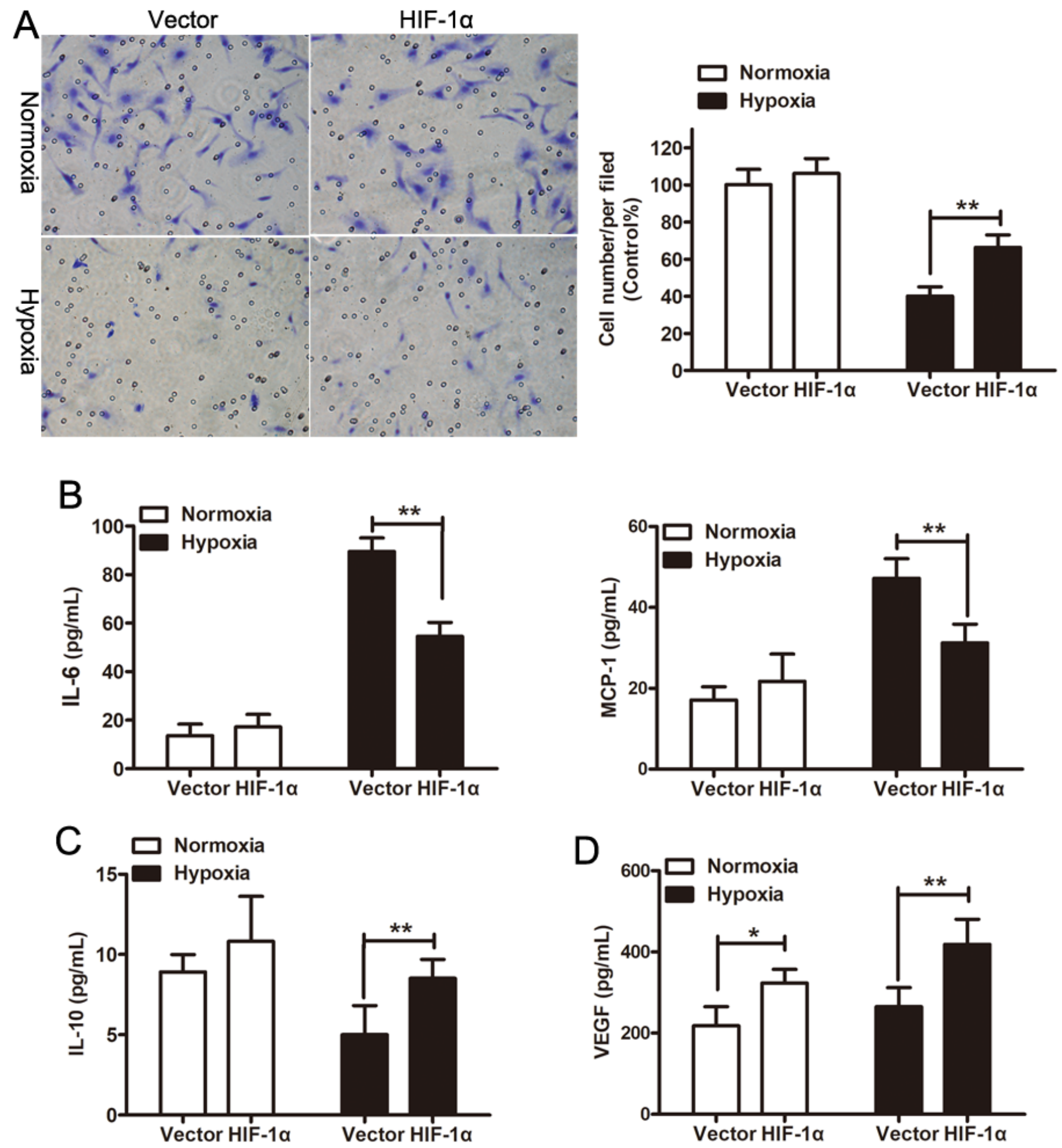

Figure 3

Exos-HIF-1a promoted HUVECs tube formation and inhibited inflammatory response under hypoxia state. (A) Representative images of the Transwell migration assay in HUVECs treated with Exos-Vector or exosHIF-1a under normoxia or hypoxia state. (B) The concentration of pro-inflammatory cytokines (IL-6 and 
MCP-1) in HUVECs supernatant was assayed by ELISA. (C) The concentration of anti-inflammatory cytokine (IL-10) in HUVECs supernatant was assayed by ELISA. (D) The concentration of angiogenic cytokine (VEGFA) in HUVECs supernatant was assayed by ELISA. **P $<0.01$.
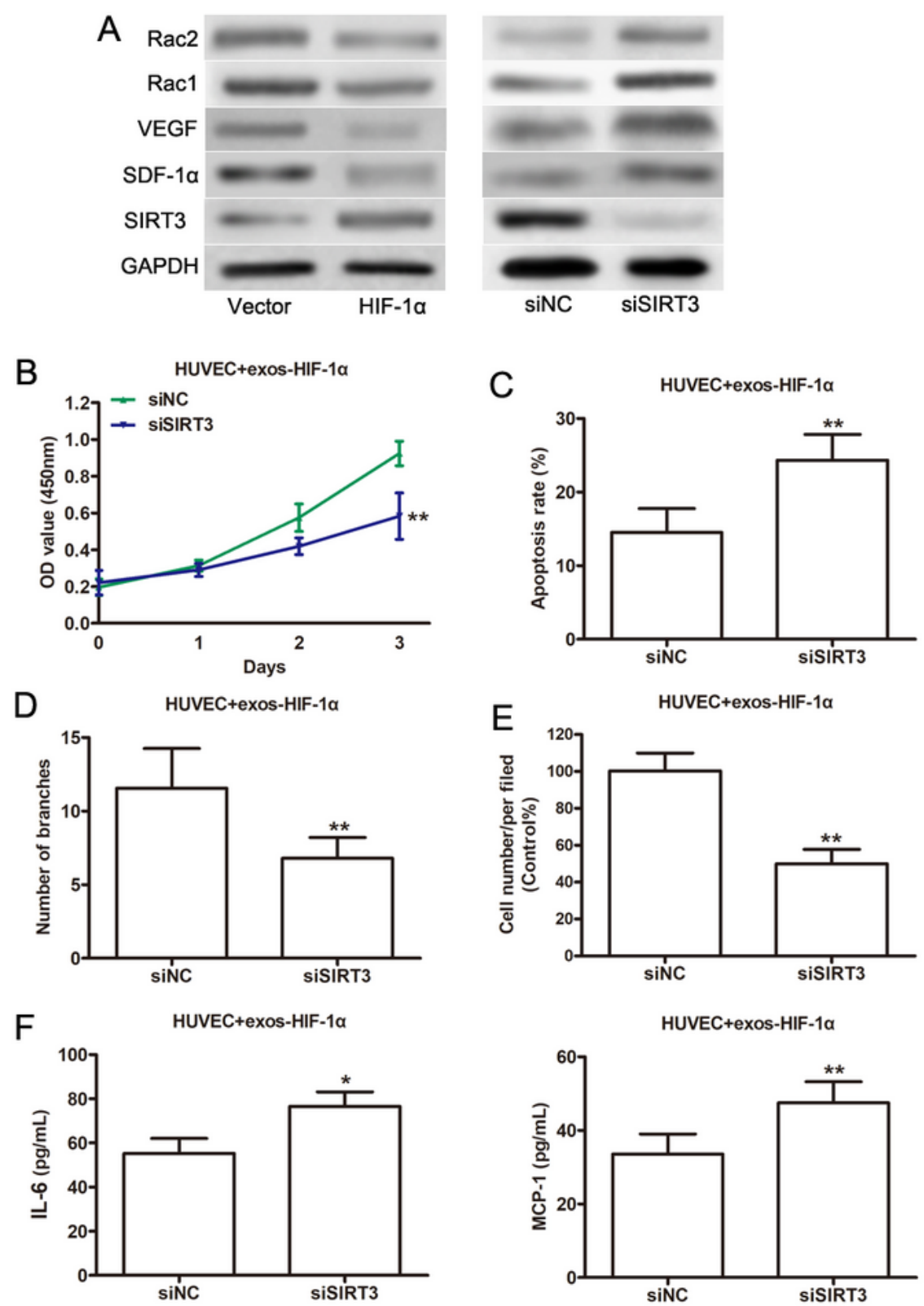

HUVEC+exos-HIF-1 $\alpha$
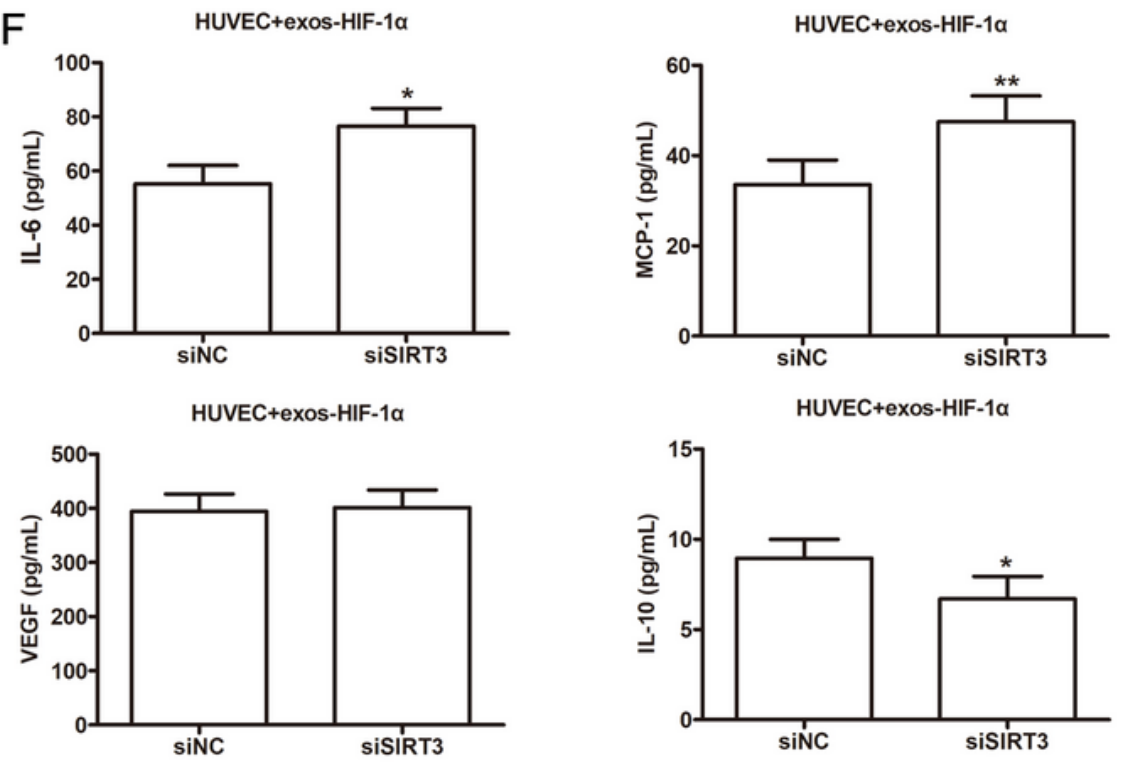

Figure 4

SIRT3 silencing attenuates hypoxia-induced dysfunction of HUVECs through the SDF-1a/Rac pathway. (A) Protein levels of SIRT3, SDF-1a, Rac1, Rac2 and VEGF was determined in HUVECs treated with exos- 
HIF-1a under hypoxia state by western blot analysis. (B) Protein levels of SIRT3, SDF-1a, Rac1, Rac2 and VEGF was determined in SIRT3 knockdown HUVECs treated with exos-HIF-1a under hypoxia state by western blot analysis. Cell viability (C), cell apoptosis (D), tube formation (E), and cell migration (F) was detected in SIRT3 knockdown HUVECs treated with exos-HIF-1 a under hypoxia state. (G) The concentration of some molecules related to pro-inflammatory cytokines (IL-6 and MCP-1), angiogenic (VEGFA), and anti-inflammatory (IL-10) were analyzed in SIRT3 knockdown HUVECs treated with exosHIF-1a under hypoxia state. ${ }^{*} \mathrm{P}<.05 ; * \star \mathrm{P}<.01$.

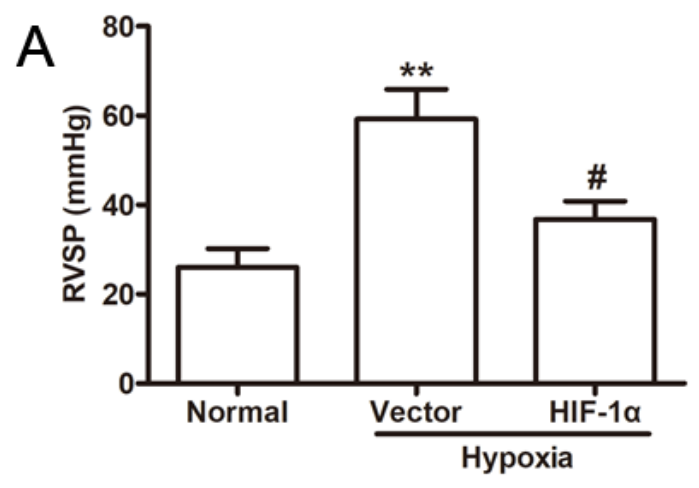

C Normal

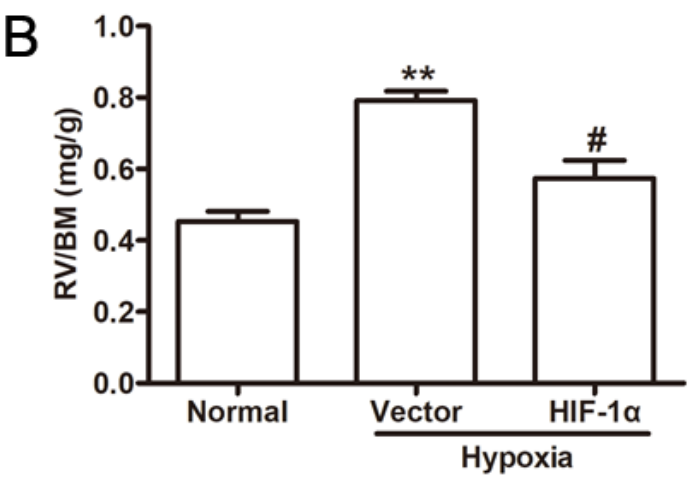

Hypoxia
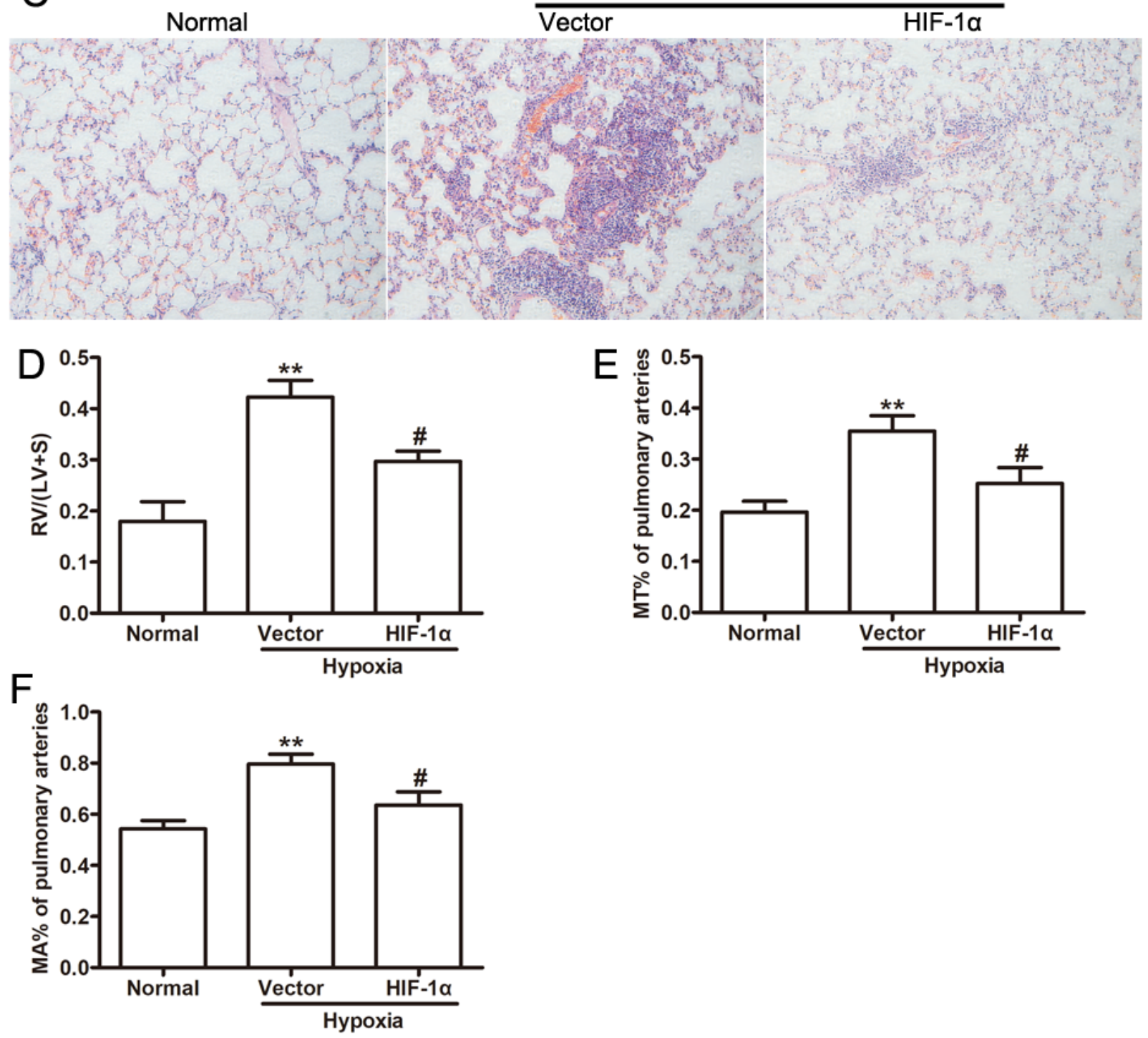


\section{Figure 5}

Exos-HIF-1a reversed pathological progression of hypoxia induced PAH in mouse models. (A)

Quantitative data of right ventricular systolic pressure (RVSP) are presented. (B and D) Quantitative data of right ventricular hypertrophy index are presented. (C) Representative images of pulmonary arteries stained with H\&E (hematoxylin \& eosin; $\times 100$ magnification). ( $E$ and $F$ ) Blinded quantitative analyses of $M T \%(E)$ and $M A \%(F)$ of peripheral pulmonary arteries were done using Image-Pro Plus. ${ }^{*} P<.01$, vs. Normal group; $\# \mathrm{P}<.05$, hypoxia for 4 weeks treated with Exos-Vector or Exos-HIF-1a. 